\title{
Estado del arte de la competencia comunicativa intercultural como nuevo enfoque metodológico en la enseñanza del inglés como lengua extranjera ${ }^{1}$
}

\author{
State of the art of intercultural communicative competence as a new methodological \\ approach in English as a foreign language teaching
}

Carlos Alberto Bernal Betancur ${ }^{2}$

Bernal B, Carlos A miradas $\mathrm{N}^{\circ} 2$ - 2019 ISSN digital $\mathrm{N}^{\circ}$ 2539-3812 Págs 66 - 81 Recepción: Febrero 11 de 2019 Aprobación: Mayo 24 de 2019 Publicación: Junio 30 de 2019

\section{Resumen}

Este artículo realiza un rastreo del estado del arte en cuanto al enfoque metodológico en la enseñanza del inglés conocido principalmente como 'La Competencia Comunicativa Intercultural'. Dicho rastreo se llevó a cabo en bases de datos disponibles en la biblioteca virtual de la Universidad Tecnológica de Pereira, la bibliografía disponible en la misma universidad y Google académico.

Los resultados evidencian dificultades en el aprendizaje del inglés. También se halló que el enfoque gramatical de enseñanza del inglés no es eficaz ni para desarrollar las competencias comunicativas de los estudiantes, ni para obtener altos puntajes en las pruebas estandarizadas. Del mismo modo se pudo evidenciar, que los docentes de inglés tienen una visión acrítica de la supremacía de este en el mundo y le otorgan mayor prestigio a los profesores nativos. Igualmente, se encontró que los gobiernos muestran un gran interés en mejorar el nivel de lengua inglesa de los estudiantes en los diferentes niveles escolares. Finalmente, se evidenció la falta de estudios relacionados al enfoque metodológico de la competencia comunicativa intercultural. En conclusión, se deben realizar investigaciones relacionadas con la implementación de enfoques comunicativos en la enseñanza del inglés que apuesten por la formación integral de usuarios de lengua competentes. Además, se deben asumir unas posiciones más críticas por parte de los docentes de inglés en cuanto al papel del idioma en el mundo.

\footnotetext{
1Estado del arte de la competencia comunicativa intercultural como nuevo enfoque metodológico en la enseñanza del inglés como lengua extranjera. Doctorado en Educación UTP. Grupo de investigación: Análisis crítico del discurso multimodal: estudios y aplicaciones transversales en ámbitos socio-académicos UTP.

2 Estudiante Doctorado en Educación UTP. Docente de inglés en Institución Educativa María Auxiliadora Dosquebradas. carlosbernal@utp.edu.co
} 
Palabras clave: Enseñanza del inglés como lengua extranjera, enfoques metodológicos para la enseñanza del inglés, el enfoque gramatical y de traducción, el enfoque comunicativo y la competencia comunicativa intercultural.

\section{Abstract}

This article sought to track the state of the art regarding the methodological approaches in teaching English known mainly as 'The Intercultural Communicative Competence'. This tracking was carried out in databases available in the virtual library of the Technological University of Pereira, the bibliography available in the same university and Google academic. The results show difficulties in learning English. It was also found that the grammatical approach to teaching English is not effective neither to develop students' communicative skills nor to obtain high scores on standardized tests. In the same way it was evident that English teachers have an uncritical vision of the supremacy of this in the world and give greater prestige to native teachers. Likewise, it was found that governments show great interest in improving the English language level of students at different school levels. Finally, the lack of studies related to the methodological approach of intercultural communicative competence was evidenced. In conclusion, investigations related to the implementation of communicative approaches in the teaching of English that bet for the integral formation of competent language users should be carried out. In addition, more critical positions must be assumed by English teachers regarding the role of this language in the world.

Keywords: Teaching of English as a foreign language, methodological approaches for teaching English, grammatical and translation approach, communicative approach and intercultural communicative competence.

\section{Introducción}

A continuación, se presentará un compendio de investigaciones doctorales a nivel nacional e internacional que comparten elementos comunes con el presente estudio 'Estado del arte de la competencia comunicativa intercultural como nuevo enfoque metodológico en la enseñanza del inglés como lengua extranjera'. El presente estado del arte busca advertir la pertinencia de las metodologías contemporáneas para la enseñanza de los idiomas, como es el caso del inglés, en especial aquellas con enfoques comunicativos e interculturales como la competencia comunicativa intercultural. Los estudios no serán presentados por orden cronológico ni por su origen locativo, sino más bien considerando un orden coherente en cuanto a la temática del estudio, partiendo de lo más general, como lo son las prácticas docentes y la educación, hasta abordar el tema clave del estudio, la competencia comunicativa intercultural como enfoque metodológico para la enseñanza del inglés como lengua extranjera. 


\section{Las prácticas docentes}

Laprácticadocente esuntema primordial dentro de las expectativas que se tienen en cuanto a las políticas educativas que promueve el gobierno; como por ejemplo: 'Colombia la más educada en el 2025', dicha política emergió en el año 2015 como resultado de la ley 1753 o Ley del Plan de Desarrollo 20142018 'Todos por un Nuevo País', cuyo mayor postulado consiste en lograr el desarrollo del país a partir de mejorar la educación en todos sus aspectos $\mathrm{y}$ niveles escolares para poder ser competitivos en el mundo globalizado del siglo XXI. A este respecto, Castro (2016) en el marco del doctorado en ciencias sociales, niñez y juventud realizó el proyecto 'Apropiación en la práctica docente del discurso de las competencias, planteamiento central de la política sectorial para mejorar la calidad educativa en Colombia' en la ciudad de Manizales, cuyo principal objetivo consistía en indagar acerca de la apropiación que tenían los docentes colombianos en su práctica pedagógica del discurso de las competencias básicas, definido por el sistema educativo colombiano representado por el Ministerio de Educación Nacional MEN.

Para cumplir con dicho objetivo, el investigador realizó una muestra intencionada de 1550 docentes, 69\% mujeres y $31 \%$ hombres con profesión docentes de educación básica primaria, básica secundariaymediapertenecientes a 22 entidades territoriales certificadas y diseminadas a lo largo de todo el territorio nacional. El instrumento que se utilizó para recoger los datos fue un cuestionario, cuyas respuestas fueron sistematizadas y analizadas estadísticamente como parte del enfoque cuantitativo de la investigación; sin embargo, debido a que el estudio fue de tipo mixto, también se realizó un análisis cualitativo que consistió en la interpretación de las preguntas abiertas del cuestionario. El objetivo de este análisis consistía en comprender la percepción de los docentes acerca de la incorporación del enfoque por competencias en las prácticas docentes y aspectos relacionados a las políticas de la calidad educativa.

El autor concluyó que efectivamente los docentes tienen información sobre el enfoque por competencias y de los estándares básicos de competencias, así como una percepción positiva de ellos. Sin embargo, su apropiación no es efectiva, principalmente por la falta de comprensión, por capacitaciones que consideran más teóricas que aplicables y porque creen que la política educativa que promueve la formación por competencias es una moda más. Finalmente, el autor concluyó que los docentes tienen poco conocimiento sobre la política de calidad, su enfoque y la especificidad de las competencias en cada área específica; así como confusiones en cuanto a la apropiación de la teoría de las competencias, lo cual se corroboró cuando al analizar los datos se pudo concluir que los docentes confundían los siguientes conceptos: logros, objetivos, metas y competencias. Siguiendo con el tema de la educación, se continúa en el siguiente estudio con lo relacionado a la acreditación escolar y la calidad de la educación. 
Estado del arte de la competencia comunicativa intercultural como nuevo enfoque metodológico en la enseñanza del inglés como lengua extranjera

Acreditación escolar y la calidad de la educación

En la línea de investigación de políticas públicas, calidad de la educación y territorio, perteneciente al doctorado en educación y sociedad de la Universidad de la Salle en la ciudad de Bogotá, Orozco (2015) realizó la investigación titulada: Diseño de un marco teórico explicativo sobre la acreditación escolar como herramienta para el mejoramiento de la calidad de la educación básica y media pública de Bogotá, D.C. La investigación estuvo enmarcada en un contexto histórico caracterizado por políticas educativas tanto a nivel local como nacional. Durante el primer periodo (2010-2014) del presidente de la república Juan Manuel Santos (denominado prosperidad para todos) se implementó la política de calidad educativa llamada 'El Camino para la Prosperidad 2010-2014'. Por otro lado, a nivel local, el alcalde Gustavo Petro Urrego durante el periodo del 2012 al 2016 dentro de su plan de gobierno denominado 'Bogotá Humana' lideró una política educativa llamada 'Construcción de Saberes, Educación Incluyente, Diversa de Calidad para Disfrutar y Aprender'.

La investigación estuvo enmarcada por tres objetivos derivados del contexto particular mencionado previamente. En primer lugar, se pretendía realizar un marco teórico explicativo acerca de la acreditación escolar como medio para mejorar la calidad de la educación en las instituciones oficiales de Bogotá. En segundo lugar, se buscaba mostrar las implicaciones, limitaciones $y$ posibilidades que surgen a partir de la implementación de un modelo de calidad en los colegios oficiales de Bogotá. Y por último, se quería identificar las percepciones sobre calidad en la educación que tenían los docentes y directivos docentes de los colegios públicos de Bogotá.

El diseño metodológico del estudio fue de tipo cualitativo enmarcado en la teoría fundamentada a partir del método de comparación constante, que consistió en una continua revisión y comparación de los datos que iban emergiendo para ir construyendo teoría, lo que permitió la formulación de nuevas categorías en base al objeto de estudio, la acreditación escolar. La información se recolectó a partir de tres instrumentos: entrevista, encuesta de percepción y análisis documental desde la revisión del muestreo teórico. La entrevista fue aplicada a investigadores y académicos de Estados Unidos de Norteamérica, Perú, Chile y Colombia que trabajan en programas de acreditación escolar y calidad educativa. Para utilizar la encuesta se seleccionaron 14 colegios oficiales con diferentes desempeños en la prueba del ICFES Saber 11 de la segunda cohorte del 2014.

Finalmente se analizaron documentos relacionados con los conceptos del marco teórico. El análisis de los datos recolectados permitió concluir que a pesar de que la acreditación escolar a nivel de primaria y secundaria es un método de evaluación no propio de la cultura colombiana, no se puede negar su importancia, eficacia y validez como factores para mejorar la educación, lo cual se ha visto reflejado desde la educación superior donde dicho 
sistema se ha implementado por un poco más de dos décadas. Igualmente, se concluyó que dicha acreditación debe ser integradora y dialogante con las políticas públicas, vinculando $\mathrm{y}$ articulando todas las instituciones educativas y sus objetivos estratégicos del proyecto educativo institucional PEI.

Otra conclusión que emergió concierne a la necesidad de avanzar en la implementación de un modelo de acreditación en la educación preescolar, básica y media como referente de la gestión escolar y las buenas prácticas educativas con el fin de mejorar la educación a nivel nacional; dicho modelo, sin embargo, debe tener estándares de calidad, procesos $\mathrm{y}$ criterios de evaluación exigentes e incluyentes que permitan el cambio y la mejora escolar, evitando que la acreditación se convierta en una práctica instrumental donde simplemente se llenen formatos y se cumplan requisitos formales. Del mismo modo, se halló que en la acreditación deben intervenir todos los estamentos de la comunidad educativa, lo cual conduciría al desarrollo social, el cambio, el progreso y la construcción de acuerdos comunes de calidad educativa, siempre y cuando esta esté acompañada de una estrategia de intervención multidisciplinar, con los recursos necesarios para su sostenimiento en el tiempo, logrando garantizar que las problemáticas que aquejan a las comunidades más vulnerables sean superadas o eliminadas totalmente.

Seguidamente, se va empezar a indagar más específicamente en el tema de la presente investigación, en lo concerniente a la enseñanza del inglés como lengua extranjera en un contexto similar al de Colombia teniendo como referente el tipo de educación (pública), la intensidad horaria semanal, el inglés como lengua extranjera obligatoria desde el nivel de básica primaria, políticas de calidad educativa y valoración del inglés como herramienta para promover el progreso de las sociedades y parte fundamental de una educación integral, y finalmente las mismas preocupaciones en lo referente al bajo rendimiento y desempeño en inglés mostrado en las pruebas censales.

\section{Desempeños en inglés}

A pesar de que en los países latinoamericanos se pudiera pensar que las dificultades en el aprendizaje del inglés como lengua extranjera es solo un asunto que incumbe a este contexto, o a naciones en vía de desarrollo, en la ciudad de Madrid, España, Vinuesa (2016) llevó a cabo un estudio que busca identificar las causas de los bajos desempeños en inglés de su población escolar. Bajo el título 'Aprendizaje de Lengua Extranjeras: Evolución Metodológica y Apuestas de Futuro', y en representación del Departamento de Economía Financiera, Contabilidad e Idioma Extranjero' de la Universidad Rey Juan Carlos. El objetivo principal del proyecto fue: identificar si es la metodología usada por los docentes $\mathrm{u}$ otros factores los que determinan el bajo desempeño de los estudiantes en las pruebas estandarizadas y el no desarrollo de la competencia comunicativa de la lengua extranjera al finalizar la educación secundaria. 
Para alcanzar los objetivos del presente estudio se seleccionó una muestra representativa de 5738 estudiantes universitarios que cursaron inglés como lengua extranjera en centros ordinarios de educación durante la primaria y la secundaria, y han ingresado al primer curso de grado de la Universidad Rey Juan Carlos, y 14603 alumnos de sexto curso de educación primaria de centros públicos bilingües de la comunidad de Madrid. La elección de los dos grupos a pesar de tener edades diferentes, se determinó por haber sido expuestos a distintas metodologías. Del mismo modo, se llevó a cabo un estudio empírico comparado entre los dos grupos cuyos datos se recolectaron en los cursos académicos de 2012-2013 y 2013-2014.

Las técnicas de recolección de datos para los alumnos universitarios fueron de tipo cualitativo, a razón de encuestas y de tipo cuantitativo mediante recopilación de datos, esto con el fin de poder determinar las percepciones de los alumnos y el nivel real de lengua. Por otro lado, los datos que se manejaron para el grupo de estudiantes bilingües se obtuvieron de la Dirección General de Mejora de la Calidad de la Enseñanza, los cuales emergieron a partir de las pruebas externas de Cambridge.

Luego de analizar los datos obtenidos del objeto de estudio, se pudo concluir que en España se le ha dado gran importancia a la enseñanza del inglés, incluso a partir de épocas remotas, iniciando con la Ley de Instrucción Pública de 1857 y culminando con la Ley Orgánica de Mejora de la Calidad
Educativa de 2013. Por el lado de la Comunidad Europea, esta también ha mostrado gran interés en la enseñanza de los idiomas mediante la publicación en 1995 del Libro Blanco: Enseñar y Aprender hacia la Sociedad del Conocimiento y en 2001 se puso a disposición de la Comunidad Europea y el mundo en general el Marco Común Europeo de Referencia para las Lenguas: Aprendizaje, Enseñanza, Evaluación. Dichos documentos buscan impulsar el aprendizaje de los idiomas extranjeros con base en propuestas metodológicas comunicativas que fortalezcan el nivel de lengua de los estudiantes. Además de dichos documentos, se pusieron en marcha programas de movilidad como: Sócrates, Comenius, eTwinning, Grundvig, Lingua o Minerva, de los cuales se benefician tanto estudiantes universitarios como profesores de todos los niveles educativos, donde estos últimos pueden aprender de manera colaborativa, difundir resultados de proyectos investigativos y compartir las buenas prácticas educativas y proyectos de innovación que incluyan el uso de las tecnologías de la información y la comunicación TIC.

En lo referente a los dos grupos de estudiantes objeto del estudio se pudo concluir que los alumnos universitarios estudiaron inglés como lengua extranjera al menos durante 12 años, siguiendo el método de la enseñanza tradicional enfocado en el aspecto formal de la lengua y la traducción descontextualizada, y aun así sus niveles de lengua al finalizar la secundaria fueron bajos. Más del $50 \%$ de dicha población solo alcanzó el nivel A2 y menos del 40\% consiguió 
un nivel B1. Por otro lado, $46 \%$ de los estudiantes bilingües de sexto grado logró el nivel B1, lo que conlleva a concluir que los alumnos de centros educativos bilingües al finalizar la secundaria llegarán a unos niveles muy altos de desempeño en las pruebas de inglés.

Igualmente, se concluyó que dos factores primordiales a la hora de poder adquirir las habilidades comunicativas en inglés son la edad de inicio en el aprendizaje (5-6 años en España) y la exposición al idioma de horas escolares semanales (3-4 horas en España), lo cual debería garantizar que al culminar la secundaria todos los estudiantes tengan al menos un nivel de lengua de B1. Sumado a lo anterior, cabe resaltar que a diferencia de otros países, en España los profesores de inglés de primaria son especialistas en la materia y no maestros generalistas.

Se encontró finalmente que el enfoque que debería ser utilizado en las aulas de clase es el de 'Content Language Integrated Learning' (CLIL) o 'Aprendizaje Integrado de Contenidos y Lengua' (AICOLE), el cual es utilizado en los centros bilingües objeto del presente estudio. Dicho enfoque ha obtenido buenos resultados en torno al desempeño de los estudiantes en pruebas estandarizadas y al desarrollo de la competencia comunicativa. AICOLE se define como un tipo de instrucción que deja de orientarse hacia el aprendizaje de la lengua, para enfocarse en el aprendizaje de contenidos de otras áreas distintas al lenguaje y como herramienta de acceso al conocimiento.

\section{Enseñanza contemporánea del inglés}

Un común denominador en tendencias educativas a nivel mundial es la enseñanza del inglés como lengua extranjera. Dicho auge viene de décadas atrás, pero se incrementó con la masificación del internet, las tecnologías de la información y la comunicación y la globalización educativa, laboral, económica y social. A este respecto, Mayoral (2016) no es ajeno a dicha tendencia en relación a la relevancia que ha cobrado el inglés a nivel global, y condujo el proyecto de tesis doctoral titulado: 'Estrategias didácticas para la enseñanza del idioma inglés a niños de preescolar: el caso de un colegio en Colima, México'. El objetivo del estudio fue el análisis a profundidad de la caracterización de las estrategias para la enseñanza del idioma inglés a niños de preescolar en un colegio privado de la ciudad de Colima, en México.

Elautorrealizóun proyectoinvestigativo de enfoque cualitativo, utilizando el método de estudio de caso debido a las características particularistas del mismo, el caso de un colegio privado de Colima con estudiantes de último grado de preescolar. Para recolectar los datos se utilizaron las técnicas de: observación participante, entrevista y análisis documental, y los instrumentos fueron: registros videográficos, registros de observación y diario de campo. La información que emergió de los datos fue organizada y codificada en categorías con el apoyo de los fundamentos teóricos de la investigación.

Derivado del análisis de los datos, emergieron dos grupos con relación a la 
práctica docente en las clases de inglés, y se consiguió elaborar un modelo educativo llamado 'Modelo Integrado de Estrategias Ecológico-Afectivas para la Enseñanza de Lenguas' (MIEEAEL), el cual se estructura en base a un ambiente de aula y de enseñanza cálido con el entorno familiar, social y académico del niño. Se encontró que las actividades que se realizan en el aula de clase son muy estructuradas y hacen parte de una dinámica muy definida con anterioridad por parte de las directivas del colegio y los docentes. De hecho, cada día viene precedido con una consigna que de alguna manera delimita el repertorio de acontecimientos que van a suceder en el día a día de la escuela.

Después de analizar los resultados que emergieron a raíz de las actividades de aula, resultaron un total de once estrategias de enseñanza y control del ambiente de aula, las cuales se originaron a partir de los códigos que compartían características comunes y se clasificaron en dos grandes grupos. El primer grupo corresponde a la didáctica, y el segundo se relaciona con actividades propias de la gestión de clase. El primer grupo se vincula a los procedimientos flexibles y reflexivos utilizados por la docente para fomentar el aprendizaje del inglés y se referenció como: 'Estrategias didácticas para la enseñanza del inglés' (EDEI). Por otro lado, las estrategias relacionadas con la normatividad, las jerarquías o niveles de autoridad, costumbres, gestión de clase y actos educativos normativos se relacionaron como: 'Estrategias para la organización y el control cálido del aula' (EPOCCA).
Concepciones acerca del inglés como lengua extranjera

Sin duda alguna, uno de los factores que más influye en el proceso de aprendizaje de una lengua extranjera, como es el caso del inglés, atañe a las concepciones que tienen los docentes acerca de la misma, y más específicamente en lo relacionado a su expansión, uso y enseñanza como lengua internacional. Moncada (2015) indagó acerca de las concepciones de un grupo de profesores de inglés de Venezuela sobre la expansión, uso y enseñanza del inglés como idioma internacional, como parte de su proyecto de tesis del Doctorado en Ciencias de la Educación de la Universidad Nacional de la Plata, Argentina.

El objetivo del estudio giró en torno a describir las concepciones de un grupo de profesores de inglés de los niveles de educación media general y educación universitaria de la ciudad de San Cristóbal, Venezuela sobre la expansión, uso y enseñanza del inglés como idioma internacional. Para cumplir con el objetivo del estudio y responder a la pregunta de investigación, la investigadora realizó un estudio de naturaleza cualitativainterpretativa con el fin de descubrir la realidad construida desde la óptica de los sujetos involucrados en el estudio. En este participaron 78 profesores de inglés de distintos colegios y universidades tanto del sector público como del privado. Los participantes fueron 43 mujeres y 35 hombres, sus edades oscilaban entre los 23 y los 70 años y su experiencia docente entre 1 y 28 años. Cabe mencionar que del 
grupo principal se seleccionaron a 9 profesores que cumplieron con un rol de informantes clave para participar en las entrevistas semi-estructuradas a profundidad. La selección de estos se basó en su disposición de tiempo, su voluntad, su conocimiento y su experiencia.

Los instrumentos que se utilizaron para la recolección de datos fueron: dos cuestionarios en línea y entrevistas semi-estructuradas en profundidad, dichos datos fueron recogidos en tres fases entre septiembre del 2014 y enero del 2015. Cabe resaltar que el análisis de datos se dio de manera integral desde el mismo momento en que se inició su recolección. Para dicho análisis, se recurrió al proceso analítico de Strauss y Corbin, que propone la generación inductiva de teoría a partir de la comparación constante de datos con el fin de crear categorías que faciliten la interpretación rigurosa de los mismos. En primer lugar se presentan los resultados relacionados con las concepciones de los profesores de inglés sobre la expansión y uso de este como idioma internacional, y al final de estos se presentó el segundo grupo relacionado con las concepciones de los docentes sobre la enseñanza del inglés como idioma internacional, en ambos casos los resultados se presentaron en tres categorías.

Los participantes concibieron el inglés como un idioma neutro y necesario para la comunicación y desarrollo personal, profesional y académico de las personas, aunque se reconoció que el auge del inglés se originó a partir del final de la segunda guerra mundial y como reconocimiento al poder militar, político y económico de naciones como Estados Unidos e Inglaterra. Los participantes no vincularon dicha supremacía con intentos de un imperialismo ideológico o cultural, y por el contrario mostraron una concepción positiva de la lengua, según ellos porque esta permite la comunicación de los individuos a nivel mundial en su rol de lengua franca. Algunos participantes tuvieron una posición crítica al afirmar que el interés del aprendizaje del inglés en Venezuela se debió a una necesidad impuesta por Estados Unidos e Inglaterra como medio o plataforma para el progreso tanto a nivel personal como social.

Los implicados en el estudio hacen referencia a las mayores y mejores oportunidades que se asocian al conocimiento y dominio del inglés, ya que este se convierte en un requisito para conseguir una vacante laboral, o sea que el inglés se entiende como una llave que abre las puertas de las oportunidades. A manera de conclusión, la autora sostiene que los participantes reflejaron poca consciencia acerca de las implicaciones socioculturales, políticas y pedagógicas relacionadas con la supremacía del inglés a nivel mundial, tampoco visualizaron que este se puede volver un instrumento para prolongar el dominio de los países más poderosos del mundo sobre el resto, un contribuyente a intensificar las desigualdades políticas, económicas y sociales.

Los participantes del estudio reflejaron concepciones positivas hacia los docentes no-nativos del inglés al enfatizar algunas de sus fortalezas 
en comparación a sus similares, los docentes nativos. Igualmente, se halló una marcada tendencia en expresar la necesidad de que los docentes nonativos tengan la oportunidad de realizar pasantías o cursos de intercambio en países angloparlantes con el fin de perfeccionar el idioma y ampliar su bagaje cultural. Otro hallazgo, tiene que ver con las experiencias negativas que han vivido en relación a la discriminación y el prejuicio, debido a su condición de docentes no-nativos fundamentadas en las concepciones del 'native speakerism', orientación tradicional que posesiona al docente nativo como el usuario ideal de la lengua, en contraposición a los maestros de inglés no-nativos, vistos como poco eficientes lingüística y culturalmente.

Las características positivas que más se resaltaron en cuanto a los docentes no-nativos fueron: el esfuerzo y la perseverancia que requiere un hablante no-nativo para aprender una segunda lengua. También, se afirmó que los docentes no-nativos son altamente competentes para ejercer su labor como docentes de inglés al contar con las competencias necesarias como lo son la lingüística y la pedagógica, sumado a la experiencia en el contexto de enseñanza. Otro aspecto favorable para los docentes no-nativos tiene que ver con el conocimiento de la lengua materna de sus estudiantes, lo cual permite realizar comparaciones entre las dos lenguas a la hora de enseñar la lengua objetivo.

Sumado a lo anterior, otro factor que se resaltó a favor de los docentes no-nativos tiene que ver con el conocimiento sociocultural de ambas lenguas, lo cual les permite realizar comparaciones, resaltar las características de ambas culturas, contextualizar la enseñanza de la lengua y actuar como mediadores culturales. Los participantes hacen hincapié en que uno de los asuntos que más promueven la discriminación laboral tiene que ver con el acento, ya que por cuestiones genéticas y propias de la biología es obviamente diferente al de los hablantes nativos. Esto se ha convertido en un estereotipo que sobrepasa los aspectos lingüísticos y perjudican en gran medida a los docentes no-nativos. Se puede concluir, que los participantes, quizás de manera inconsciente, siguen sometidos a las ideas propias del 'native speakerism'. Del mismo modo, se concluye que hubo algunas contradicciones por parte de los docentes objeto del estudio, cuando se declaraban fortalecidos y competentes tanto en el plano lingüístico como en el pedagógico para enseñar el inglés, pero al mismo tiempo mostraron su cercanía a las ideas propias del 'native speakerism' al asegurar que un complemento y necesidad en sus hojas de vida laborales, tendría que ser la oportunidad de viajar a un país angloparlante para poder perfeccionar la lengua y asimilar y aprender sobre la cultura de dichos países.

En cuanto a las metodologías de enseñanza también se hizo claro que existía una percepción acrítica de las mismas, ya que los participantes insistían en que estas estaban enfocadas en el desarrollo de las competencias comunicativas y estaban libres de propósitos ideológicos de poder. Los 
participantes también manifestaron que promueven desde sus clases espacios para socializar temas socioculturales y políticos concernientes a los intereses de los estudiantes, lo anterior va en contravía de los enfoques comunicativos que pretenden insertar a los aprendices en las culturas extranjeras de manera no reflexiva y sin mostrar sus verdaderos intereses, hecho que va en línea contraria a las propuestas pedagógicas que promueven la reflexión crítica de los sujetos basadas en las realidades sociales propias de los contextos particulares de cada individuo, y que les permita ser los actores protagónicos de su propia historia libres de cualquier tipo de imposición u opresión.

En conclusión, los docentes de inglés deben formar futuros usuarios de inglés que tengan la capacidad de analizar críticamente el contenido cultural de la lengua estudiada, con relación a su propia cultura, lo cual reafirmaría su sentido de pertenencia a la comunidad de la que hace parte activa, y los ayudaría a convertirse en 'hablantes interculturales'. A continuación se trae a colación un estudio que está relacionado con la competencia cultural en torno a la enseñanza del inglés en el aula de clase.

\section{El componente cultural en los libros de texto de inglés}

Varón (2011) tituló su investigación: 'Componentes culturales en el aprendizaje del inglés: un estudio sobre los libros de texto contemporáneos usados para la enseñanza y el aprendizaje del inglés en el nivel de básica secundaria en Colombia', la cual fue llevada a cabo en la ciudad de Pereira. La autora invita a la reflexión en cuanto a lo que significa el uso de una lengua extranjera con fines comunicativos y trae a colación aspectos poco relacionados en el pasado como lo son los prejuicios y los estereotipos, la otredad y la formación en valores como el respeto y la tolerancia hacia los demás, el reconocimiento de las diferencias y necesidades, pero que resultan fundamentales en la comunicación intercultural. A partir de este contexto, la autora se interesa por indagar acerca de las construcciones culturales que tienen lugar en los libros de texto, ya que estos son las herramientas usadas con mayor frecuencia para la enseñanza y el aprendizaje del inglés en la actualidad.

Así mismo, la autora planteó los siguientes objetivos investigativos: contribuir en el campo de la didáctica de lenguas extranjeras en cuanto a la relación lengua y cultura, y la inclusión de contenidos culturales en los currículos escolares; discutir la pertinencia del libro de texto en el aula de clase como herramienta para el aprendizaje del inglés; contribuir con el desarrollo profesional de los docentes de inglés y despertar su interés por el componente cultural desde una posición crítica; conocer los supuestos teóricos que argumentan la enseñanza de aspectos culturales en el aula de clase; estudiar la temática cultural en los contextos de enseñanza y aprendizaje de lenguas e identificar la naturaleza de los contenidos y temáticas culturales en una muestra de libros usados en Colombia. 
En cuanto a la metodología, la autora ubicó el estudio en la categoría de ensayo, de análisis temático interdisciplinar, de tipo inductivo $\mathrm{y}$ enmarcado en las técnicas generales de análisis de contenido en libros de texto. El análisis de contenido de la investigación se identificó como 'product oriented research' el cual se interesa en el producto mismo, ya sea como constructor ideológico, transmisor cultural o como instrumento pedagógico. El objetivo de este tipo de análisis consiste en mejorar la legibilidad, la inteligibilidad, la fidelidad, la objetividad y la eficacia de los textos analizados y de las futuras producciones. Dicha etapa de la investigación se enfocó en analizar y describir las diferentes maneras en que se presentó el contenido cultural de los libros de texto usados por los estudiantes colombianos para el aprendizaje del inglés como lengua extranjera, asumiendo de entrada que los libros de texto marcan la identidad de las culturas estudiadas.

Los libros de texto seleccionados para el estudio fueron contemporáneos posteriores al año 2004 y de nivel de secundaria. La mayoría de los mismos fueron producidos en países angloparlantes y unos pocos lo fueron en Colombia. La investigación se enfocó en el libro manejado por el estudiante denominado 'Student's book', el cual se utiliza mayormente con carácter individual. Para la recolección de la información se elaboró una rejilla diseñada con seis categorías de análisis: los objetivos propuestos por el libro de texto, los temas y el tratamiento a la información cultural provista, las actividades culturales, los personajes en el libro de texto, el modelo o los modelos fonológicos ejemplificados a través de los audios y la evaluación del conocimiento cultural.

Los resultados del análisis fueron: en cuanto a los objetivos culturales, no se encontraron propuestas concretas y explícitas; en lo referente a los temas culturales, los que más se presentan son viajes a nuevos lugares, el entretenimiento y la ocupación del tiempo libre, también se encontró una marcada tendencia a mostrar un mundo sin problemas, obviando así asuntos y problemáticas sociales incomodos de discutir. Se muestran imágenes con paisajes bonitos y famosos y personajes ficticios disfrutando de viajes y lugares paradisiacos. A lo anterior, se concluyó que el libro de texto se ofrece como un catálogo turístico y el estudiante se concibe como un turista futuro en potencia.

En cuanto a las actividades culturales, se destaca que todos los libros del corpus seleccionado desarrollan pedagogías activas, donde se concibe a un estudiante activo que hace tareas mediante las cuales desarrolla la competencia comunicativa y mejora la competencia lexical y la gramatical. También, se encontró que ninguno de los textos del corpus define explícitamente sus actividades como culturales; sin embargo, todos ellos proponen actividades que se pueden clasificar de ese tipo, como instrucciones para consolidar el conocimiento cultural anticipándose a problemas de comunicación intercultural e información sobre variados grupos culturales sin limitarse a los del 
círculo interno y actividades basadas en aspectos actitudinales y afectivos promoviendo la otredad y la empatía; en lo referente a los personajes, estos juegan un rol muy importante en los libros de texto de lenguas extranjeras, ya que estos proporcionan las visiones culturales y se afianzan o derriban ideologías.

Se presentaron personajes ficticios transitorios $y$ otros no ficticios correspondientes en la mayoría de los casos al mundo anglo. Los personajes ficticios se caracterizaban por los perfiles se seres felices y exitosos tanto en el hogar como en el trabajo; igualmente, se incluyen personajes que representan ciudadanos cosmopolitas que realizan viajes internacionales, sin problemas sociales o económicos y cuyas preocupaciones recaen en las posibilidades para acceder a experiencias nuevas de entretenimiento. Pasando al modelo fonológico, se halló que todos los textos del corpus incluyen únicamente modelos a seguir norteamericanos $\mathrm{y}$ británicos en sus audios; finalmente, en cuanto a la evaluación cultural, se encontró el elemento más negativo de la investigación y es lo concerniente a la total ausencia de objetivos y de instrumentos de evaluación del componente cultural.

La implementación de la Competencia Comunicativa Intercultural en el aula de inglés

En la última década se han venido implementando estudios que guardan relación con la competencia comunicativa intercultural (CCI) en el aula de clase de inglés como lengua extranjera; y a este respecto, Guadamillas (2014) decidió incursionar en una investigación relacionada con el uso de la poesía, la canción, el teatro y el cómic como pilares para la enseñanza del inglés enmarcado en la CCI. La autora llevó a cabo su tesis doctoral en Toledo, España, titulada 'El desarrollo de la competencia comunicativa intercultural a través de recursos creativos, análisis de la poesía, el teatro y el cómic como materiales didácticos en la enseñanza del inglés como lengua extranjera'.

Los objetivos generales de la investigación fueron: conocer en qué grado los docentes de lengua inglesa conocen, saben y usan recursos y materiales creativos en el aula, tales como la poesía, la canción, el teatro y el cómic, la opinión que tienen los docentes en cuanto a la pertinencia o no de dichos recursos, analizar los materiales que contienen los manuales de inglés de niveles intermedios y que contienen elementos artísticos, los niveles, criterios y competencias propuestas por el Marco Común Europeo de Referencia (MCER) dirigidos al desarrollo de la CCI y proponer criterios para analizar los manuales de lengua inglesa, para así conocer el potencial comunicativo e intercultural de sus actividades.

Para cumplir con los objetivos propuestos, la autora optó por la investigación cualitativa, con la cual pretendió conseguir descripciones exhaustivas de una realidad concreta en torno a las actividades y los materiales utilizados en el aula de clase de carácter comunicativo e intercultural que faciliten el aprendizaje del inglés como 
lengua extranjera. Los instrumentos que se utilizaron para recolectar los datos fueron encuestas distribuidas a cuarenta profesores de nivel intermedio de secundaria, las cuales buscaban comprobar con qué frecuencia dichos maestros utilizaban materiales como canciones, poesía, cómic y teatro en el aula de clase con fines de aprendizaje de la lengua inglesa. Del mismo modo, se analizaron manuales de las editoriales internacionales más usadas para verificar si estos tenían relación con el aspecto creativo, lo visual, lo musical o lo dramático. Por último, se utilizaron cuestionarios estructurados que constaban de quince preguntas que hacían referencia a las prácticas docentes y a los materiales innovadores utilizados para la enseñanza del inglés $\mathrm{y}$ al conocimiento del MCER en lo que concierne al enfoque comunicativo.

Los resultados arrojaron que el $45 \%$ de los centros de estudio apoyan poco el uso de materiales artísticos y solo un $14 \%$ de los encuestados afirmó que reciben mucho apoyo por parte de sus instituciones. Por otro lado, se halló que el $60 \%$ de los profesores utilizan el juego de roles o el teatro como herramienta primordial para la enseñanza del inglés con un enfoque comunicativo y no de manera aislada o esporádica. Sin embargo, solo el 15\% de los encuestados admite usar bastante el cómic, mediante su lectura o creación como componente fundamental para la enseñanza comunicativa de la lengua, frente a un $62 \%$ que dice usarlo poco. En concordancia con lo anterior, tan solo un $13 \%$ de los docentes, constató que hace uso frecuente de la poesía como herramienta para practicar o promover actividades de pronunciación, mientras un $30 \%$ asegura nunca haber usado la poesía para tal fin. Habiéndosele preguntado de manera general a los maestros acerca de su capacidad creativa e innovadora en el desarrollo de las actividades que promueven el aprendizaje del inglés en el aula, tan solo un $23 \%$ de ellos aseguró ser muy creativos.

En conexión con el anterior resultado, se indagó acerca de la libertad que los maestros tenían en sus instituciones educativas para utilizar recursos creativos y metodologías modernas para la enseñanza de lenguas, a lo que un $82 \%$ de ellos, contestó tener mucha libertad para tal fin. Así mismo, a la pregunta acerca de la exigencia o demanda por parte de los superiores, jefes de área o coordinadores académicos para implementar nuevas metodologías, innovación y creatividad en el aula, un $42 \%$ de los encuestados contestó que dicha exigencia es poca, y un $20 \%$ afirmó que nunca los exhortan en tales aspectos.

En cuanto al enfoque comunicativo como marco de referencia para la enseñanza de lenguas propuesto por el MCER, un $78 \%$ de los docentes dijo conocerlo mucho, y tan solo un $2 \%$ de los mismos afirmó no conocer nada al respecto. En cuanto a la formación que poseían los docentes para poder aplicar el teatro, la poesía y el cómic en el aula de clase con fines de aprendizaje, el $54 \%$ de los docentes dijeron tener poca formación al respecto. Los resultados en cuanto a la forma como dichos docentes aprendieron el inglés en términos de la utilización de materiales 
creativos como teatro, poesía o cómic fueron muy bajos, tan solo un $23 \%$ de ellos, afirmaron que aprendieron con la implementación de dicho tipo de materiales en el aula durante su etapa como estudiantes.

Del mismo modo, se concluyó que aun cuando los docentes son conscientes del potencial y utilidad de los recursos creativos, su implementación en el aula es muy baja, y la razón de lo anterior recae en la falta de tiempo o una pobre preparación o formación para explorar e implementar dichos recursos, de los cuales el más utilizado en el aula de clase es el juego de roles, mientras el menos usado es la poesía; lo anterior, aplica de igual manera para los manuales analizados. Así mismo, se pudo concluir que la habilidad de lengua más trabajada fue el 'speaking' la cual se implementó en parejas y grupos pequeños, mientras que el 'reading' y el 'writing' se trabajaron poco. En cuanto a la relación entre los manuales y el MCER, se encontró que todas las secuencias didácticas estaban relacionadas con el marco en cuanto al desarrollo de las competencias de lengua. Finalmente se pudo identificar que el manual 'New Cutting Edge' fue el que mejor se adaptó a los veinte criterios de análisis estudiados.

El estado del arte planteado en los anteriores párrafos producto de un rastreo de tesis doctorales nacionales e internacionales relacionadas con la enseñanza del inglés y metodologías contemporáneas, permite deducir la necesidad de utilizar dichas metodologías en el aula de clase de inglés; en especial, la Competencia
Comunicativa Intercultural, ya que es esta la que más se adapta al momento actual que vive la sociedad como resultado de los avances tecnológicos y la globalización, que requiere de ciudadanos que puedan interactuar y comunicarse en inglés, pero que también reconozcan desde el plano lingüístico y cultural a ese otro con el que se relacionan.

\section{Referencias bibliográficas}

Castro, H. (2016). Apropiación en la práctica docente del discurso de las competencias,

planteamiento central de la política sectorial para mejorar la calidad educativa en

Colombia (tesis de doctorado). Universidad de Manizales, Manizales, Colombia.

Guadamillas, M.V.(2014).El desarrollo de la competencia comunicativa e intercultural a

través de recursos creativos, análisis de la poesía, el teatro y el comic como

materiales didácticos en la enseñanza del inglés como lengua extranjera (tesis de

doctorado). Universidad de Castilla-La Mancha, Toledo, España.

Mayoral, P.J. (2016). Estrategias didácticas para la enseñanza del idioma inglés a niños de

preescolar: el caso de un colegio en 
Colima, México (tesis de doctorado). Instituto

tecnológico y de estudios superiores de España.

Occidente, Jalisco, México.

Moncada, B.S. (2015). Concepciones de un grupo de profesores de inglés de Venezuela

sobre la expansión, uso y enseñanza del inglés como idioma internacional

(tesis de doctorado). Universidad Nacional de la Plata, La Plata, Argentina.

Orozco, J.C. (2015). Diseño de un marco teórico explicativo sobre la acreditación escolar

comoherramienta para el mejoramiento de la calidad de la educación básica y

media pública de Bogotá, D.C. (tesis de doctorado). Universidad de la Salle,

Bogotá, Colombia.

Varón, M.E. (2011). Componentes culturales en el aprendizaje del inglés: un estudio sobre

libros de texto contemporáneos usados para la enseñanza y el aprendizaje del

inglés en el nivel de básica secundaria en Colombia (tesis de doctorado).

Universidad Tecnológica de Pereira, Pereira, Colombia.

Vinuesa, V. (2016). Aprendizaje de lenguas extranjeras: evolución metodológica $y$ apuestas de futuro (tesis de doctorado). Universidad Rey Juan Carlos, Madrid, 